Recepción: 15/01/2019

Aceptación: 19/02/2019

Publicación: 05/04/2019

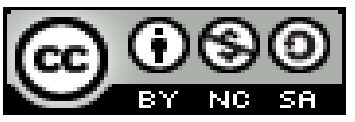

Ciencias de la educación

Artículo de investigación

\title{
EI teatro de sombras para la enseñanza de la historia ecuatoriana
}

\author{
The shadow theater for the teaching of ecuadorian history
}

O teatro das sombras para o ensino da história do equador

\author{
Cecilia del Carmen Costa-Samaniego ${ }^{\text {I }}$ \\ cecilia.costa@unl.edu.ec \\ Brayan Ronald Romero-Pinto II \\ brromerop@unl.edu.ec
}

Correspondencia: cecilia.costa@unl.edu.ec

I Magíster en Administración para el Desarrollo Educativo, Abogada, Doctora en Ciencias de la Educación, Licenciada en Ciencias de la Educación en la Especialidad de Ciencias Sociales, Profesora de Educación Media en la Especialidad de Ciencias Sociales. Docente Universidad Nacional de Loja. Loja, Ecuador.

II Licenciado en Ciencias de la Educación Mención Educación Básica, Docente Escuela Particular San Andrés. Loja, Ecuador. 


\section{Resumen}

El presente trabajo estuvo orientado a conocer la efectividad del uso del teatro de sombras como estrategia didáctica para mejorar la enseñanza de la historia ecuatoriana en los estudiantes de sexto grado "C", de la UEP San Gerardo, periodo septiembre 2017 - julio 2018, de la ciudad de Loja. Esta investigación se enmarca en el tipo de estudio descriptivo, con un enfoque mixto (cuanti-cualitativo) y el diseño fue cuasi-experimental; los métodos utilizados fueron el analítico, sintético, heurístico, estadístico, descriptivo, inductivo, deductivo, histórico y observacional. Se diseñó y empleó un taller metodológico sobre el correcto uso de esta innovadora estrategia. La aplicación del mismo permitió una mejora del $47 \%$ en el dominio del conocimiento sobre la historia ecuatoriana, además de desarrollar la creatividad y trabajo cooperativo, traduciéndose esto en la factibilidad del uso de esta estrategia dentro del ámbito educativo, para solventar las dificultades de los estudiantes durante el desarrollo del conocimiento histórico en las clases de Estudios Sociales. Al finalizar el taller se aplicó una prueba de conocimientos, en la cual se notó que los estudiantes se sintieron más motivados por aprender y el clima de la clase mejoró favorablemente, produciendo un incremento en los aprendizajes. Se recomienda utilizar esta estrategia en las demás áreas del conocimiento y buscar su adaptación en los demás subniveles de la Educación Primaria.

Palabras clave: enseñar; estrategia didáctica, historia ecuatoriana; teatro de sombras.

\section{Abstract}

The present work was oriented to know the effectiveness of the use of shadow theater as a didactic strategy to improve the teaching of Ecuadorian history in the sixth grade $\mathrm{C}$ students of the San Gerardo UEP, September 2017 - July 2018, in the city of Loja. This research is framed in the type of descriptive study, with a mixed (quantitative-qualitative) approach and the design was quasi-experimental; The methods used were analytical, synthetic, heuristic, statistical, descriptive, inductive, deductive, historical and observational. A methodological workshop on the correct use of this innovative strategy was designed and used. The application of the same allowed a $47 \%$ improvement in the domain of knowledge about Ecuadorian history, in addition to developing creativity and cooperative work, translating this into the feasibility of using this strategy within the educational field, to solve the difficulties of the students during the 
development of historical knowledge in Social Studies classes. At the end of the workshop a knowledge test was applied, in which it was noted that the students felt more motivated to learn and the classroom climate improved favorably, producing an increase in learning. It is recommended to use this strategy in the other areas of knowledge and seek their adaptation in the other sublevels of Primary Education.

Keywords: teaching; didactic strategy; ecuadorian history; shadow theater.

\section{Resumo}

O objetivo deste estudo foi determinar a eficácia do uso do teatro de sombras como estratégia de ensino para melhorar o ensino da história equatoriana no grau sixth- "C", a UEP San Gerardo, período de setembro de 2017 - julho 2018, a cidade de Loja. Esta pesquisa enquadra-se no tipo de estudo descritivo, com abordagem mista (quantitativa-qualitativa) e o delineamento foi quase experimental; Os métodos utilizados foram analítico, sintético, heurístico, estatístico, descritivo, indutivo, dedutivo, histórico e observacional. Uma oficina metodológica sobre o uso correto desta estratégia inovadora foi projetada e usada. A sua aplicação permitiu uma melhoria de 47\% no domínio do conhecimento sobre a história do Equador, bem como desenvolver a criatividade e trabalho em equipe, traduzindo esta avaliando a possibilidade de usar esta estratégia no sector da educação, para enfrentar as dificuldades estudantes durante o desenvolvimento do conhecimento histórico nas aulas de Estudos Sociais. No final do workshop, foi aplicado um teste de conhecimento, no qual se observou que os alunos se sentiam mais motivados para aprender e o clima de sala de aula melhorava favoravelmente, produzindo um aumento na aprendizagem. Recomenda-se utilizar essa estratégia nas demais áreas do conhecimento e buscar sua adaptação nos demais subníveis da Educação Básica.

Palavras-chave: ensinar; estratégia didática, história equatoriana; teatro de sombras.

\section{Introducción}

Las Ciencias Sociales se conforman por un grupo de disciplinas científicas, cuyo objeto de estudio está vinculado a las actividades y el comportamiento de los seres humanos; por lo que, las Ciencias Sociales, analizan las manifestaciones de la sociedad, tanto materiales como simbólicas. La importancia de las ciencias sociales radica en la necesidad de estudio y examinación de las sociedades y conductas humanas por medio de asignaturas que faciliten analizar las interacciones y el desarrollo cultural en el mundo; de ahí que las ciencias sociales tributan con conocimientos 
profundos, que van más allá de una experiencia inmediata y ayudan a expresar y a advertir el funcionamiento de las sociedades, las causas y posibles soluciones a diversas problemáticas establecidas en la misma.

En la actualidad, los Estudios Sociales afrontan el desafío de concentrar nuevas dimensiones producidas en la vida de los seres humanos (violencias, problemas ambientales, culturales, políticos, etc.), que desbordan los marcos interpretativos de las disciplinas; por ello, requieren de la integración de saberes y miradas transdisciplinares, que brinden respuestas contextualizadas a los problemas y requerimientos de la sociedad y junto con ello la aplicación de estrategias didácticas adecuadas, para que el conocimiento sea aprehendido de manera fácil y significativa por parte de los estudiantes.

Sin embargo, las condiciones sociales, culturales y políticas del Ecuador, son el reflejo de la crisis mundial del sistema capitalista; y a ello se aúna la falta de presupuesto educativo, la aplicación de procesos de enseñanzas monótonos y tradicionales, la falta de capacitación docente, la escasa motivación de éste, la inexistencia de recursos didácticos, entre otras situaciones que para nada aportan a la tarea académica, haciendo inclusive difícil cumplir el rol docente de manera eficaz y eficiente.

Hoy en día en la mayoría de la población escolar, la enseñanza de la historia ecuatoriana ha significado una de las disciplinas más complejas durante el proceso de enseñanza en el sistema ecuatoriano, todo esto debido a la complejidad de sus características; a las nuevas necesidades de la sociedad educativa actual; y, la falta de utilización de estrategias didácticas innovadoras por parte de los docentes, problemas que han sido evidenciados durante el proceso investigativo, realizado con los estudiantes del sexto grado paralelo "C" de la Unidad Educativa Particular San Gerardo, durante el periodo escolar comprendido entre septiembre 2017- julio 2018.

A la luz de esta situación, se coincide con Romero (2015) quien aporta que las clases de historia no necesariamente tienen que ser largas y aburridas. Esta asignatura, en especial, se presta para realizar un gran número de actividades que permiten al maestro interactuar con sus estudiantes para que aprendan de una manera más efectiva. De ahí que, en la investigación realizada se plantearon actividades que permitieron diagnosticar el estado actual de las dificultades en la 
enseñanza de la asignatura de Estudios Sociales y su incidencia en el aprendizaje de la historia ecuatoriana en los estudiantes motivo de nuestra investigación; esto llevó directamente a determinar los referentes teóricos y metodológicos a través de la literatura científica sobre el teatro de sombras, como estrategia didáctica, la cual facilitó establecer la propuesta de un cronograma de actividades mediante la aplicación del taller metodológico. Ejecutadas las actividades, se pudo validar el impacto de la propuesta de la aplicación de la estrategia el teatro de sombras y su aporte para mejorar el aprendizaje de la historia ecuatoriana.

La explicación teórica se hizo con base en dos variables: La enseñanza de la historia ecuatoriana y el teatro de sombras como estrategia didáctica, cada variable tiene subtemas como la definición de estrategia metodológica; problemas en el aprendizaje; su utilidad; la didáctica; incidencia en la escuela; fines educativos de la enseñanza de la historia; objetivos didácticos; y, el método histórico; así mismo en la variable dependiente encontramos la definición; su efecto como estrategia didáctica; los elementos esenciales; la repercusión de su aplicación en la escuela; y, el papel que cumplen los actores dentro del proceso de enseñanza-aprendizaje.

\section{Materiales y Metodología}

Esta investigación se enmarca en el tipo de estudio descriptivo, con un enfoque mixto (cuanticualitativo) y el diseño fue cuasi-experimental; los métodos utilizados fueron el analítico, sintético, heurístico, estadístico, descriptivo, inductivo, deductivo, histórico y observacional, mismos que sirvieron para fundamentar el problema, establecer el marco teórico, operativizar el análisis de los resultados, hasta llegar a las conclusiones y recomendaciones. Se utilizaron las técnicas como la encuesta, entrevista y prueba escrita e instrumentos como el cuestionario de preguntas y de base estructurada y un test de conocimiento de 10 preguntas sobre hechos históricos del Ecuador; y, como no podía faltar la aplicación del taller metodológico, con el uso de la estrategia del teatro de sombras, motivo esencial de nuestra investigación. Así mismo se realizó el análisis e interpretación de la información obtenida de los cuestionarios dirigidos a estudiantes y docentes, lo que permitió conocer las dificultades en el proceso de enseñanza de esta disciplina.

Luego de la aplicación del taller metodológico como estrategia alternativa, se llegó a la formulación de conclusiones, la principal de ellas resultó ser que la utilización del teatro de sombras como estrategia didáctica es muy importante dentro de la enseñanza de la historia 
ecuatoriana porque permite fortalecer su creatividad, el desarrollo de la psicomotricidad fina, la aplicación del trabajo cooperativo y el desarrollo de la expresión corporal de los estudiantes, lo que desencadena en la consecución de aprendizajes significativos y aplicables en su vida cotidiana. Como recomendaciones a los docentes que apliquen variadas estrategias innovadoras, particularmente el teatro de sombras, para que a la par que cumplen su labor académica, faciliten el aprendizaje de sus estudiantes; y, a las autoridades de la institución que realicen constantes evaluaciones sobre el dominio de la historia del Ecuador, de tal forma que se pueda tener conocimiento sobre si existe o no un progreso en el conocimiento de los estudiantes, ya que de no ser así, se podría aplicar los correctivos correspondientes en el proceso de formación.

\section{Objetivos}

Diseñar una propuesta alternativa basada en el teatro de sombras como estrategia didáctica para mejorar la enseñanza de la historia ecuatoriana en los estudiantes del sexto grado paralelo "C" de la Unidad Educativa Particular San Gerardo, periodo septiembre 2017 - julio 2018.

\section{Específicos}

- Establecer los referentes teóricos y metodológicos a través de la literatura científica sobre el teatro de sombras como estrategia didáctica para mejorar el aprendizaje de la historia ecuatoriana.

- Diagnosticar el estado actual de las dificultades en la enseñanza de la asignatura de estudios sociales y su incidencia en el aprendizaje de la historia ecuatoriana

- Proponer un cronograma de actividades que se ejecutarán en relación a la aplicación de la técnica del teatro de sombras para mejorar el aprendizaje de la historia ecuatoriana

- Ejecutar las actividades planteadas sobre el teatro de sombras para mejorar el aprendizaje de la historia ecuatoriana

- Validar el impacto que han tenidos las actividades propuestas sobre teatro de sombras para mejorar el aprendizaje de la historia ecuatoriana

\section{Referentes teóricos}

- La enseñanza de la historia ecuatoriana 


\section{Definición}

La Historia como materia educativa nos ofrece un relato del pasado. Los niños/as deben de comprender que las acciones, decisiones, valores, etc. del pasado influyen en el presente y en el futuro (Espinosa y Gregorio, 2018, p. 194). Dentro del ámbito educativo, la historia como disciplina educativa tiene un papel muy relevante en la formación integral de los estudiantes, para lo cual es de vital importancia que estos comprendan la influencia que tienen las acciones, decisiones y valores del pasado en la realidad actual y por supuesto en el futuro.

Por su parte, Rats (2017) refiere que la historia es una de las disciplinas escolares más complicadas y difíciles de enseñar, tanto por su propia naturaleza de ciencia formal, como por el uso y abuso de los poderes públicos y políticos que alteran su normal desarrollo. Sumándole a esto, la comodidad que tienen los docentes al no aplicar la pedagogía al momento de enseñar esta disciplina a sus estudiantes. La enseñanza de la historia es de vital importancia en el desarrollo integral de todo niño, dentro del marco social, cultural y personal. Es así que hoy en día en el Ecuador, la enseñanza de esta disciplina resulta un problema presente en todo el sistema educativo nacional, debido a que hasta en la actualidad se pretende enseñar de manera tradicional, lo que provoca disparidades en el proceso educativo del contenido histórico del estudiante.

Para que el proceso de enseñanza sea efectivo, es importante que se cumpla con ciertos parámetros: fijarse un objetivo o una meta a cumplir; proponer lo que se quiere lograr en el niño, es decir las destrezas con criterio de desempeño, plantearse las estrategias didácticas y metodológicas pertinentes para abordar el contenido, hacer uso de recursos para la enseñanza, verificar si las estrategias aplicadas han sido las correctas o las más acertadas para desarrollar en los estudiantes un aprendizaje significativo.

En ese orden, se asume que dentro de las Ciencias Sociales tienen cabida un sinnúmero de disciplinas, que al igual que la historia, su enseñanza contribuye enormemente en la formación integral del ser. Tal como refiere González (2012, p. 26): "El estudio de la Historia no debe concebirse como un hecho aislado sino como parte de un proceso que para su análisis requiere de otras ciencias, por lo tanto, la debida correlación permitirá mayor facilidad para su enseñanza." Al igual que la sociología, psicología, o incluso la geografía, la historia es una disciplina muy 
compleja y de suma importancia para el niño y su bienestar académico, además de que cada una de estas disciplinas resulta complementaria en el dominio de las demás.

\section{Enseñanza de la historia}

Dentro del proceso de enseñanza de la historia como tal, el docente tiene un papel muy importante, ya que es el encargado de direccionar este proceso y seleccionar las estrategias, métodos, materiales y recursos más idóneos para garantizar el aprendizaje significativo de los niños. Clark, Pearson y Carretero (citados en Lima, 2016) mencionan que la enseñanza de la historia es una actividad interactiva y de carácter procesual, que depende en gran medida de la manera en que el docente ejerce, concibe, planifica, ejecuta y reconsidera su participación dentro de este proceso. El docente debe estar preparado para este reto y para ello necesita una formación constante, contar con el apoyo necesario de sus familiares, autoridades y todos quienes lo acompañan.

La enseñanza de la historia es en sí un problema de gran relevancia; es un obstáculo en sí mismo, ya que la mayoría de docentes desconocen la importancia y trascendencia de la misma, por lo tanto, se hace evidente que se carece de los recursos didácticos apropiados para su enseñanza (Romero, 2015). Debido a los múltiples errores que se han cometido en la enseñanza de la historia a lo largo del tiempo, los alumnos tienen una idea errónea de la importancia que tiene hoy en día aprender historia en su vida. "El alumnado considera que la asignatura de Historia, y la propia historia, no necesita ser comprendida sino memorizada" (Prats, 2017, p.17). Visión que está equivocada, ya que para que un aprendizaje sea significativo y en especial, el conocimiento histórico, es muy importante que el niño se apropie de los contenidos, logrando así una verdadera comprensión, y dejando a un lado la memorización.

\section{La propuesta: diseño del taller pedagógico}

El taller pedagógico se configura en una variedad de concepciones educativas, estrategias didácticas y se nutre por la diversidad de criterios que producen un intercambio de ideas entre los participantes. Además, el taller pedagógico se asume como un proceso integrador de actividades de enseñanza y aprendizaje conducentes a formar en los participantes una actitud científica, crítica y reflexiva. (Alfaro \& Badilla, 2015, pp. 86-87). El taller pedagógico debe planificarse con la intención de que los participantes construyan el conocimiento, aprovechando el potencial 
académico y la experiencia adquirida en los diferentes contextos educativos, en el que se desenvuelven.

\section{EI taller como forma del trabajo metodológico para la educación desarrolladora}

Existen variadas definiciones que se dan con respecto a lo que es taller metodológico pero se considera que las mejores son aquellas que incluyen las funciones que se han de desarrollar dentro del mismo. D. Calzado (1998, p. 1) señala la diversidad de actividades pedagógicas a las que se le denominan taller, categorizándose también como método, procedimiento, técnica y forma de organización del proceso pedagógico." Se coincide con la definición que sobre taller metodológico ofrece el autor, puesto que a la par que lo define, también lo diferencia del resto de las formas de organización del trabajo que debe realizarse, considerándolo como una forma de organización que concuerda con la concepción problematizadora y desarrolladora de la educación, en la medida en que a través de él se trata de salvar la división que se produce entre teoría y práctica, entre producción y trasmisión de conocimientos, habilidades y hábitos de investigación y docencia.

"El análisis de esta definición permite comprender que el taller ofrece efectivamente mayores alternativas a los estudiantes en cuanto a las funciones didácticas que puede cumplir" (D. Calzado, 1998, p.2). Con ello existe coincidencia en relación a que en el taller metodológico se dan condiciones que permiten, entre otras, que se propicie el trabajo en equipo o grupal; que se logre, en correspondencia con su preparación, vincular la teoría con la práctica en donde se discutan problemáticas científicas y de carácter metodológico que elevan la labor del profesional, así como su preparación, sirviendo finalmente como vía para la generalización y validación de experiencias docentes que eleven la calidad y resultados en la preparación del profesional de los docente y su aplicación efectiva durante el proceso de enseñanza aprendizaje con los estudiantes.

\section{El teatro de sombras como estrategia didáctica}

La didáctica es considerada como el arte de enseñar a todos y cada uno de los estudiantes que conforman un grupo de aprendizaje, de diferentes niveles cognitivos y de acuerdo a su etapa evolutiva. Es así que el teatro de sombras como estrategia didáctica puede definirse como un componente necesario para profundizar en el conocimiento y vivenciar de forma eficaz y eficiente la expresión de nuestro propio cuerpo, mismo que debe ser adaptado a las posibilidades 
de cada estudiante, teniendo en cuenta las posibles dificultades o necesidades de estos, Barba (citado en Rubio, 2015).

\section{Metodología}

Los métodos utilizados y aplicados pertinentemente durante el proceso de investigación y que aportaron de manera efectiva en ésta tarea indagativa fueron:

El observacional, mismo que posibilitó identificar la situación problemática y sus causas, todo esto gracias a su carácter descriptivo y vinculación con el paradigma denominado investigación cualitativa; analítico, empleado en la interpretación de los resultados, además de permitir identificar y clasificar en categorías los datos recolectados para determinar su situación actual; sintético, utilizado para sintetizar la información recolectada, facilitando la comprensión del objeto de estudio y para redactar las conclusiones y recomendaciones; inductivo, se lo utilizó para el estudio y análisis de los hechos particulares y deducir criterios para plasmar las conclusiones; deductivo, utilizado durante el estudio de la relación de las variables y al tratar de verificar la información de la investigación de campo con los datos empíricos y con base teórica.

Así mismo, se aplicaron los métodos: Hermenéutico, utilizado en el planteamiento del marco teórico y para la interpretación de los resultados obtenidos del trabajo realizado, facilitando la interpretación de la teoría es decir, la información recabada; histórico, el cual fue usado para determinar el origen de las variables, además de su evolución tomando como base estudios previos relacionados a la temática estudiada y que han sido correctamente avalados, para la realización de un adecuado contraste, constante en la verificación del cumplimiento de los objetivos planteados; estadístico, utilizado para organizar los resultados cuantitativos y las representaciones gráficas de los mismos que obtuvieron al desarrollar la investigación; descriptivo, permitió plantear la problemática a partir de la recolección minuciosa de información conceptual y documental, utilizada en la elaboración de los objetivos.

\section{Técnicas e instrumentos utilizados}

Para el trabajo de campo se utilizaron las siguientes técnicas e instrumentos:

La Encuesta, aplicada a los estudiantes del sexto grado paralelo "C" de la UEP San Gerardo, esta técnica tuvo como instrumento un cuestionario de siete preguntas, y permitió conocer las causas 
de las dificultades en la enseñanza de la historia del país así como la carencia de la utilización del teatro de sombras como una estrategia que aporta al conocimiento significativo de los estudiantes; la entrevista, aplicada a las cuatro docentes de aula de la población investigada, cuyo instrumento fue un guión conformado por nueve preguntas de base estructurada. A través de esta técnica se logró recoger información con respecto a las causas de las dificultades en la enseñanza de esta disciplina y las razones por las cuales no se hace uso del teatro de sombras como estrategia didáctica innovadora para la enseñanza de la historia ecuatoriana; la prueba escrita, aplicada a los 16 estudiantes motivo de la investigación, teniendo como instrumento un cuestionario de 10 preguntas, el cual posibilitó determinar el nivel de conocimientos que tienen en relación a la historia ecuatoriana y la existencia de las dificultades en la enseñanza de la historia.

\section{Procedimientos para el desarrollo de la investigación}

Con respecto a la organización de los datos experimentales, se tomó como elementos organizados a las variables de cada una de las hipótesis específicas que orientaron el desarrollo del trabajo de investigación; en lo que respecta a la tabulación de la información, se aplicó la estadística descriptiva para la cuantificación y observación del porcentaje de los datos; en la representación gráfica de los datos experimentales, se elaboró cuadros y gráficos que facilitaron la comprensión visual de la información, utilizando para ello los programas informáticos Microsoft y Excel; para el análisis e interpretación de los datos, se tomaron en consideración los planteamientos teóricos expuestos en cada una de las categorías explicadas en el marco teórico de la investigación, contrastada en los resultados de la investigación de campo.

En lo relacionado a la verificación de las hipótesis específicas, la vía empírica posibilitó el proceso de discusión sobre cada uno de los indicadores investigados, y se pudo contrastar la información empírica con los planteamientos del marco teórico, en donde, a partir de los datos más significativos, se procedió a la verificación de cada una de las hipótesis específicas planteadas; y, finalmente en lo que tiene que ver a la formulación de conclusiones, se tomó en cuenta la formulación de objetivos generales y específicos así como, los principales resultados de las hipótesis específicas que orientaron la investigación, para garantizar coherencia lógica entre todos los componentes del proceso investigativo desarrollado. 


\section{Resultados y Discusión}

Los resultados de los instrumentos aplicados a los diferentes actores de la comunidad educativa de la UEP San Gerardo, de la ciudad de Loja, se contrastaron con la fundamentación teórica, lo que permitió establecer importantes resultados que se evidencian en las respuestas dadas a las preguntas planteadas en los diferentes instrumentos de investigación y que ha servido como argumento para el desarrollo del presente trabajo investigativo. La enseñanza-aprendizaje dentro del aula, es un proceso que busca lograr en cada estudiante aprendizajes significativos y perennes en su vida; dentro de dicho proceso realizado en el área de Estudios Sociales, se puede evidenciar que muchos de los estudiantes no logran adquirir los aprendizajes al mismo ritmo que sus compañeros y por ende, la mayoría de ellos no dominan la historia del Ecuador, por lo que al no poseer un conocimiento histórico básico, resulta difícil conseguir que aprendan los hechos mucho más complejos, con respecto a la historia del país.

De esta manera, realizar clases de refuerzo académico sobre la historia del país es necesario y oportuno, pero no solo eso, si los estudiantes no lograron ese conocimiento con clases ordinarias, se debe buscar una estrategia que permita llamar la atención del estudiante y facilitar su aprendizaje, por lo que utilizar estrategias didácticas innovadoras, especialmente el teatro de sombras consideramos que permitirá que los estudiantes aprendan de forma lúdica y que ese aprendizaje sea sólido y significativo.

Los resultados obtenidos en la investigación en relación a cada objetivo planteado son los siguientes:

Primer objetivo. Fundamentar teóricamente la estrategia del teatro de sombras, mediante la búsqueda de una adecuada bibliografía científica, para mejorar la enseñanza de la historia ecuatoriana en los estudiantes del sexto grado, paralelo "C" de la UEP San Gerardo, periodo septiembre 2017- julio 2018.

El primer objetivo específico se cumplió con efíciencia, empezando con la búsqueda de información en las diferentes fuentes disponibles, lo que permitió construir la revisión literaria con información confiable, que aporten a nuestro bagaje de conocimientos. Esta información se encuentra enmarcada en las dos variables de investigación; la primera, la enseñanza de la historia 
ecuatoriana y la segunda referida al teatro de sombras como estrategia didáctica. También se puede evidenciar su cumplimiento en la metodología, en el análisis e interpretación de resultados y en la elaboración del taller pedagógico y su valoración.

Segundo objetivo. Diagnosticar la eficiencia del proceso de enseñanza de la historia ecuatoriana en los estudiantes del sexto grado "C" de la UEP San Gerardo, periodo septiembre 2017- julio 2018, mediante la aplicación de la estrategia del teatro de sombras para mejorar la enseñanza. Para el cumplimiento de este objetivo se aplicó un cuestionario, cuyos resultados se presentan a renglón seguido:

1. ¿Cuáles de las siguientes estrategias didácticas utiliza la docente durante la clase de Historia del Ecuador?

Tabla 1

\begin{tabular}{lcc}
\hline Alternativas & \multicolumn{2}{c}{ Estudiantes } \\
& f & \% \\
\hline Resúmenes & 15 & 27 \\
Exposiciones & 16 & 29 \\
Lectura comentada & 7 & 13 \\
Debates & -- & -- \\
Trabajos en grupo & 15 & 27 \\
Teatro de sombras & -- & -- \\
Preguntas y respuestas & -- & -- \\
Collages & -- & -- \\
Dramatizaciones & -- & -- \\
Cuadros sinópticos & 2 & 4 \\
Otros (maquetas, lectura comprensiva, salidas de campo) & & -- \\
TOTAL & & $\mathbf{1 0 0}$
\end{tabular}

Fuente. Encuesta aplicada a los estudiantes del sexto grado paralelo "C" de la Unidad Educativa Particular San Gerardo.

Elaboración. Costa y Romero (2018) 


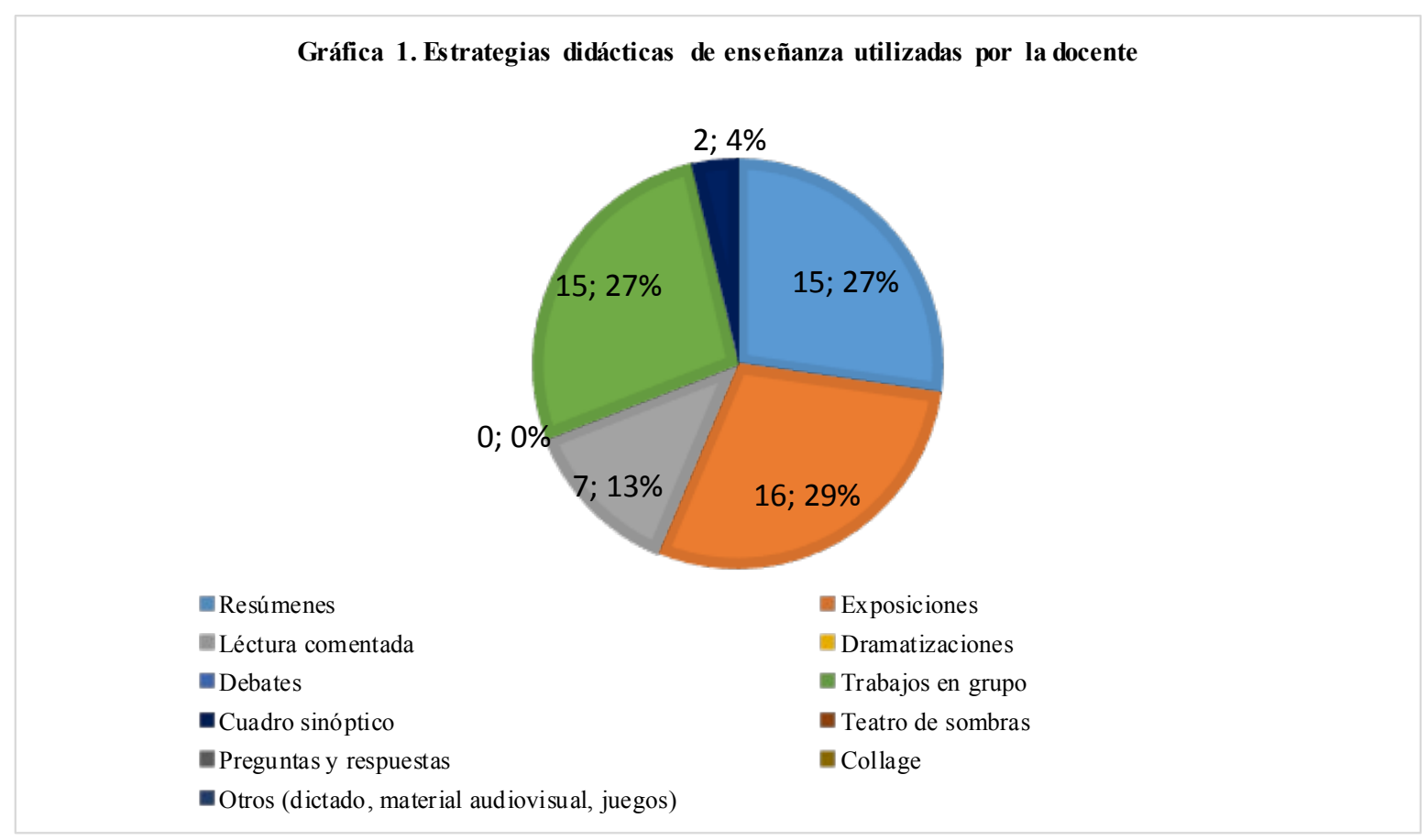

Gráfica 1. Resultados sobre Estrategias Didácticas de enseñanza utilizadas por el docente

\section{Análisis e interpretación}

De los 16 estudiantes encuestados que comprende la totalidad del paralelo, señalan que las estrategias que más utiliza la docente para la enseñanza de la historia ecuatoriana son los resúmenes, las exposiciones y los cuadros sinópticos, seguidos de otros no menos importantes; mientras que el teatro de sombras carece de pronunciamiento alguno. A lo largo de la historia han existido un sinnúmero de estrategias didácticas para la enseñanza de la historia ecuatoriana, tales como resúmenes, cuadros sinópticos o exposiciones que, aunque en su tiempo eran muy utilizados, a causa de sus efectos, hoy en día ya no tienen esa garantía, debido a las características actuales de la población escolar, como lo demuestran los estudiantes encuestados. Estos resultados son contrastados con el aporte dado por el autor, quien manifiesta que para garantizar al aprendizaje histórico se debe introducir nuevas estrategias que posibiliten una mejora en el rendimiento académico de los estudiantes.

Entre las estrategias didácticas para enseñar Estudios Sociales tenemos: resúmenes, exposiciones, trabajos en grupo, cuadros sinópticos, preguntas y respuestas, collage, debates, teatro de sombras, lectura comentada y dramatizaciones. En su ejercicio docente ¿Cuáles de ellas usted aplica 
durante el proceso de enseñanza - aprendizaje en sus estudiantes, en lo que respecta a la historia ecuatoriana?

Como docente aplico un sinnúmero de estrategias didácticas, como el collage, resúmenes, debates, trabajos en grupo, ya que son las más idóneas para tratar estas temáticas. De la respuesta podemos deducir que es importante que como docentes se conozcan los distintos tipos de estrategias didácticas que se pueden usar en el aula y sobre todo saber apreciar el potencial que tiene cada una y el contexto en el cual pueden ser utilizadas. El criterio vertido por la docente refleja de forma evidente el poco conocimiento que posee, mencionando inclusive las técnicas más tradicionales y que poco aportan al conocimiento de los estudiantes, por lo visto desconoce la estrategia del teatro de sombras, misma que al ser aplicada aporta conocimientos significativos en los estudiantes.

Tercer objetivo. Proponer la aplicación de la estrategia del teatro de sombras fundamentada en un adecuado conocimiento científico, para mejorar la enseñanza de la historia ecuatoriana en los estudiantes del sexto grado de la UEP San Gerardo, periodo septiembre 2017- lulio 2018.

El cumplimiento de este objetivo, se basó en la elaboración de un taller metodológico sobre la utilización de la estrategia del teatro de sombras para la enseñanza de la historia ecuatoriana, el cual fue fundamentado de acuerdo a las necesidades de la población escolar, mismas que se vieron reflejadas en los resultados del diagnóstico, gracias a lo que se obtuvo de la siguiente pregunta:

2. ¿Considera que las estrategias didácticas utilizadas por la docente le benefician en el aprendizaje de la historia ecuatoriana?

Tabla 2

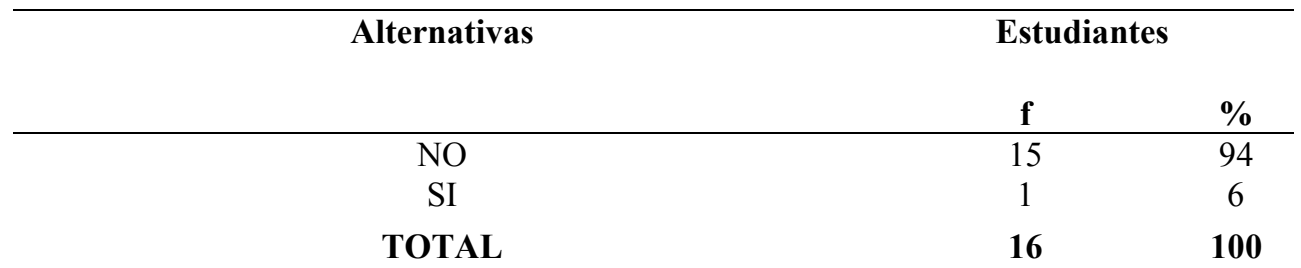

Fuente. Resultados obtenidos de la encuesta aplicada a los estudiantes del sexto grado paralelo "C" de la Unidad Educativa Particular San Gerardo.

Elaboración. Costa y Romero (2018) 


\section{Gráfica 2. Eficiencia de las estrategias didácticas}

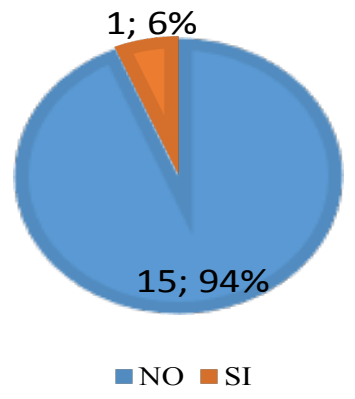

Gráfica 2. Resultados de la eficiencia de las estrategias didácticas

\section{Análisis e interpretación}

De los 16 estudiantes encuestados, 15 de ellos que reflejan el 94\% indican que las estrategias que usa la docente no son beneficiosas para su aprendizaje; y, un estudiante que equivale al $6 \%$ expresa que esas estrategias sin son beneficiosas para su aprendizaje. La mayoría de estudiantes manifiestan que las estrategias didácticas que utiliza la docente no son beneficiosas para aprender sobre la historia del país, de ahí que señalan que sus clases son monótonas y cansadas, además de que los pocos conocimientos que se adquieren no son duraderos. Los autores mencionados anteriormente, hablan sobre la importancia del uso de estrategias adecuadas durante el proceso de enseñanza aprendizaje para desarrollar habilidades y destrezas que garanticen la enseñanza de la historia. Asimismo, se presentan los resultados de la aplicación de la pregunta: ¿Le gustaría aprender la historia ecuatoriana a través de la estrategia didáctica del teatro de sombras? De lo cual se obtuvo lo siguiente:

\section{3. ¿Le gustaría aprender la historia ecuatoriana a través de la estrategia didáctica del teatro de sombras?}

Tabla 3

\begin{tabular}{lcc}
\hline Alternativas & \multicolumn{2}{c}{ Estudiantes } \\
& F & \% \\
\hline SI & 16 & 100 \\
NO & - & - \\
TOTAL & $\mathbf{1 6}$ & $\mathbf{1 0 0}$ \\
\hline
\end{tabular}

Fuente. Resultados obtenidos de la aplicación de $\boldsymbol{l a}$ Encuesta aplicada a los estudiantes del sexto grado paralelo "C" de la Unidad Educativa Particular San Gerardo.

Elaboración. Costa y Romero (2018) 


\section{Gráfica 3. Aprender la historia ecuatoriana usando el teatro de sombras}

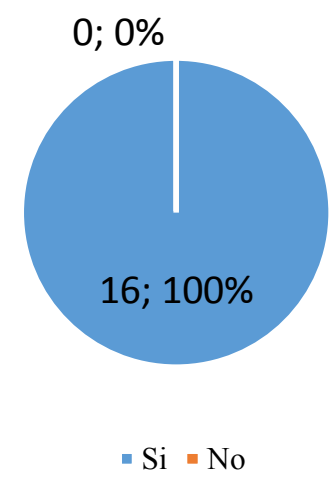

Gráfica 3. Resultados sobre aprender la historia ecuatoriana usando de sombras

\section{Análisis e interpretación}

De los 16 estudiantes encuestados que corresponde al $100 \%$, en su totalidad manifestaron de forma rotunda, que sí les gustaría aprender la historia ecuatoriana través del teatro de sombras, como estrategia didáctica innovadora, ya que consideran que es algo nuevo que les servirá para ampliar su bagaje de conocimientos. En lo que concierne a esta pregunta, todos los estudiantes están de acuerdo con aprender la historia ecuatoriana a través de una nueva estrategia didáctica, como es el teatro de sombras, que les facilitará en gran medida su aprendizaje, además de contribuir con su creatividad y el trabajo en equipo, lo cual es fundamentado en el aporte dado por el autor antes citado, quien comenta que esta estrategia didáctica resulta una alternativa sumamente útil dentro de la labor docente, ya que a más de facilitar la comprensión de contenidos también contribuirá con el desarrollo de la creatividad y la expresividad de los estudiantes. Cuarto objetivo. Aplicar la estrategia del teatro de sombras, mediante el desarrollo de un taller pedagógico, para mejorar la enseñanza de la historia ecuatoriana en los alumnos del sexto grado de la UEP San Gerardo, periodo septiembre 2017- julio 2018.

El objetivo se logró en su totalidad, al aplicar la estrategia o propuesta alternativa planteada y gracias a la predisposición de estudiantes y la docente por participar en el taller metodológico. Heras (citado en Pallares, López y Bermejo, 2014) piensa que, para garantizar el éxito en el proceso de implementación de la estrategia del teatro de sombras, por ende se considera que es muy importante que exista un adecuado clima de cohesión grupal en el aula de clase, además de un aumento de confianza entre todos los participantes y por supuesto un ambiente de seguridad 
digno para todos, lo que permitirá que todo el trabajo sea más productivo y que se obtenga el máximo provecho a los aprendizajes adquiridos.

Este taller estuvo conformado por nueve actividades, trabajando representación de relatos históricos del país, como la "Batalla del Pichincha"; "La Desmembración Territorial", entre otros temas, cuyos argumentos fueron reformulados y adaptados por los propios estudiantes, para luego ser puestos en escena, mediante una representación teatral haciendo uso de la estrategia del teatro de sombras, las cuales permitieron a la docente considerar su uso en futuras temáticas a ser abordadas durante el proceso de enseñanza y a los estudiantes los motivó a aprender sobre la historia del Ecuador de manera interactiva, lúdica y divertida.

Quinto Objetivo. Validar la estrategia del teatro de sombras mediante el desarrollo de un taller pedagógico para mejorar la enseñanza de la historia ecuatoriana en los alumnos del sexto grado de la UEP San Gerardo, periodo septiembre 2017- julio 2018.

Este objetivo se cumplió satisfactoriamente, su valoración se realizó a lo largo de todo el proceso de aplicación del taller metodológico, haciendo uso de un sinnúmero de técnicas e instrumentos de evaluación como listas de cotejo y escalas valorativas. Al finalizar el taller se aplicó una prueba de conocimientos, la misma que también fue utilizada al inicio del trabajo de investigación, obteniendo resultados verdaderamente positivos, donde los estudiantes tuvieron un aumento del $47 \%$ sobre el dominio de la historia ecuatoriana, además también se notó que los estudiantes se sintieron más motivados por aprender y el clima de la clase mejoró favorablemente, produciendo un incremento en los aprendizajes. Por lo tanto, se valora positivamente la propuesta del taller metodológico sobre la estrategia del teatro de sombras para mejorar la enseñanza de la historia ecuatoriana.

Con éste breve análisis y discusión de resultados se señala que: La primera hipótesis fue rechazada, ya que el $100 \%$ de docentes manifiestan que conocen lo que son las estrategias didácticas. Son docentes que han tenido una adecuada formación; la segunda hipótesis fue comprobada, el $94 \%$ de los estudiantes manifiestan que la docente no utiliza las estrategias idóneas para impartir la disciplina de historia, lo cual repercute de gran manera en la formación histórica del estudiante; la tercera hipótesis fue comprobada, ya que la totalidad, es decir el $100 \%$ 
de las docentes mencionan que, aunque conocen vagamente lo que es el teatro de sombras, su totalidad no utiliza esta estrategia en sus clases de historia, aunque también presentaron predisposición por participar en el taller pedagógico sobre el correcto uso de esta estrategia innovadora; y, la cuarta hipótesis fue comprobada, ya que el 100\% de los estudiantes presentaron una mejora en su dominio de la historia del Ecuador, una vez que la docente hizo uso de esta estrategia, donde se usa como su principal herramienta didáctica al teatro de sombra.

\section{Conclusiones}

Del diálogo mantenido con estudiantes y docentes del plantel, luego de haber conocido las ventajas de aprendizaje que brinda la aplicación de ésta estrategia didáctica como es el teatro de sombras, se deduce que la misma es empleada de manera pertinente y oportuna pudiendo evidenciar la eficacia derivada de su aplicación, obteniendo como producto un aprendizaje significativo de la historia ecuatoriana en los estudiantes, dado que de su aplicación se alcanzaron las siguientes conclusiones:

- Se logró diseñar una propuesta alternativa sostenida en el interés y la necesidad que demostraron tanto los docentes como los estudiantes por aplicar el teatro de sombras como estrategia didáctica, para mejorar el proceso de enseñanza-aprendizaje de la historia ecuatoriana.

- Se consiguió argumentar de forma teórica las variables de la presente investigación, mediante la utilización de bibliografía y literatura adecuada, lo que permitió determinar la importancia de la aplicación del teatro de sombras como estrategia didáctica, dentro del proceso de enseñanza de la historia ecuatoriana.

- Se concretó la aplicación de la estrategia didáctica del teatro de sombras, mediante el desarrollo de un taller metodológico, en donde los participantes se familiarizaron con los procedimientos de esta estrategia, a efectos de poder mejorar el proceso de enseñanzaaprendizaje de la historia ecuatoriana.

- Se validó la efectividad de la aplicación del taller metodológico sobre el uso de la estrategia didáctica del teatro de sombras, y se evidenció en los resultados de la aplicación de un post test a los estudiantes motivo de ésta investigación, con una progresión considerable. 


\section{Recomendaciones}

- Que las autoridades y docentes del plantel educativo investigado se informen de manera bibliográfica/literaria sobre la importancia que tiene y las bondades que se derivan de la aplicación de la estrategia didáctica el teatro de sombras, dentro del proceso de enseñanza de la historia ecuatoriana.

- Autoridades y docentes, multipliquen las veces que sean necesarias con los actores del proceso educativo del plantel investigado, el taller metodológico de la estrategia didáctica del teatro de sombras, a efectos de mejorar el proceso de enseñanza-aprendizaje de la historia ecuatoriana.

\section{Referencias Bibliográficas}

Alfaro, A. \& Badilla, M., 2015. El taller pedagógico, una herramienta didáctica para abordar temas alusivos a la Educación Ciudadana. Costa Rica: CIDICER.

Calzado, D., 1998 El taller: una alternativa de forma de organización para los Institutos Superiores Pedagógicos. Tesis de Maestría. ISP Enrique José Varona. Inédita.

Lima, L., 2016. El aprendizaje de la Historia: una propuesta de estrategias de comprensión lectora. History and History teaching, (42) 12-34.

Rubio, S. y Bermejo, A., 2015. El teatro de sombras. Valladolid - España: Universidad de Valladolid.

González, M. T., 2012. La enseñanza de la historia en la educación básica. México: Historia.

Prats, J., 2001. Enseñar historia y geografía. Principios básicos. Histodidáctica, (3).

Prats, J. (2017). Retos y dificultades para la enseñanza de la Historia. Barcelona- España: Milenio.

Prats, J. y Santacana, J., 2010 Enseñar Historia: notas para una didáctica renovada, España Mérida: Junta de Extremadura.

Romero, P., 2015 Importancia de la enseñanza de historia. LA PRENSA. Recuperado de https:/impresa.prensa.com/Importancia-ensenanza-historia_0_4267073359.html 